\title{
A Microfluidic Aptamer-Based Sensor for Detection of Mer- cury (II) and Lead (II) Ions in Water
}

\author{
Wei-Hao Huang ${ }^{1}$, Ruo-Yin Wu ${ }^{1}$, Ko-Li Yeh ${ }^{1}$, Van-Phung Mai ${ }^{1}$ and Ruey-Jen Yang ${ }^{1, *}$ \\ 1 Department of Engineering Science, National Cheng Kung University, Tainan, Taiwan. \\ * Correspondence: rjyang@mail.ncku.edu.tw; Tel.: +886-6-2757575 Ext. 63343; Fax: +886-6-2766549
}

\begin{abstract}
Heavy metal contaminants have serious consequences for the environment and human health. Consequently, effective methods for detecting their presence, particularly in water and food, are urgently required. Accordingly, the present study proposes a sensor for the detection of mercury $\mathrm{Hg}$ (II) and lead $\mathrm{Pb}$ (II) ions using graphene oxide (GO) as a quenching agent and aptamer solution as a reagent. In the proposed device, the aptamer sequences are labeled by FAM and HEX fluorescent dyes, respectively, and are mixed with 500 ppm GO solution in a microfluidic device. The presence of $\mathrm{Hg}(\mathrm{II})$ and $\mathrm{Pb}$ (II) ions is then detected by measuring the change in the fluorescence intensity of the GO/aptamer suspension as the aptamer molecules undergo fluorescence resonance energy transfer (FRET). The experimental results show that the aptamer sensors have a linear range of 10 250 nM (i.e., 2.0 50 ppb) for $\mathrm{Hg}$ (II) ions and 10 100 nM (i.e., 2.1 20.7 ppb) for Pb(II) ions. Furthermore, the limit of detection is around $2 \mathrm{ppb}$ for both metals, which is significantly lower than the maximum limits of $6 \mathrm{ppb}$ and $10 \mathrm{ppb}$ prescribed by the World Health Organization (WHO) for $\mathrm{Hg}(\mathrm{II})$ and $\mathrm{Pb}(\mathrm{II})$ in drinking water, respectively.
\end{abstract}

Keywords: Aptamer; Fluorescence Resonance Energy Transfer; Heavy Metal Ions; Graphene Oxide; Microfluidic Device; Sensor.

\section{Introduction}

Pollution caused by human activity is a serious worldwide problem nowadays; with massive environmental, financial and health ramifications. Among the many different types of contaminants found in water, land and the air, heavy metal ions pose a particularly significant risk to both the environment and human health. Consequently, effective methods for detecting trace amounts of heavy metal ions with high sensitivity and good selectivity are urgently required [1]. Mercury $(\mathrm{Hg})$ is one of the most toxic heavy metals in common use and can cause a wide variety of unpleasant and dangerous disorders, including lung damage, diarrhea, nausea, and permanent organ damage [2]. Lead $(\mathrm{Pb})$ is also an extremely common heavy metal, and is used extensively throughout the construction, water, electrical and battery industries. However, the presence of lead in water is harmful to human health even at low exposure levels and can result in delays in physical and mental development, together with serious attention and learning deficits [3].

During the past few years, many methods have been reported for heavy metal ion detection. Among the various techniques which have been proposed, aptasensors are particularly attractive due to their high affinity, high specificity and wide versatility as a biological receptor for many different binding targets [4,5]. Furthermore, aptamers can be easily mass-produced, purified, and tailored to the capture of particular targets through the design of specific sequences [5]. For example, aptamers with a thymine (T)-rich sequence can be used to form a mismatched complex $\left(\mathrm{T}-\mathrm{Hg}^{2+}-\mathrm{T}\right)$ with $\mathrm{Hg}^{2+}$ ions by changing 
the configuration to a hairpin-shape [6]. Similarly, aptamers with a guanine (G)-rich sequence can be made to readily bond with $\mathrm{Pb}^{2+}$ ions to form a G-quadruplex structure [7]. Aptasensors are compatible with many standard detection methods, including colorimetric, fluorescent and electrochemical signals. Among these methods, electrochemical signals are particularly sensitive to the resistance change caused by the combination of the aptamer molecules with the target analytes. However, the pre-treatment process required for the electrode is complicated and time-consuming [8,9]. Accordingly, recent studies have focused increasingly on the use of attenuated total reflection surface enhanced infrared absorption spectroscopy (ATR-SEIRAS) as an alternative sensing technique [10]. Fluorescent sensors have many practical advantages as a sensing tool, including a facile operation, a high sensitivity and easily detectable signals. Most fluorescent sensors are based on the fluorescence resonance energy transfer (FRET) effect, in which energy transfer occurs between an excited donor fluorophore and an acceptor via a non-radiative mechanism without the absorption or emission of photons [11]. Graphene and graphene oxide (GO) have attracted growing interest in the sensing field in recent years since aromatic ring structures based on graphene and GO surfaces readily adsorb and combine with many biological molecules (including aptamers) through a $\pi-\pi$ stacking mechanism and electron transfer. The resulting aromatic ring structures thus provide an outstanding platform for fluorescence-based sensing [12-15]. Notably, graphene and its derivatives are beneficial not only in preventing the aptamers from changing their configuration before binding to the target, but also serve as excellent quenchers with minimal background interference. Thus, GO/aptamer sensors have unparalleled advantages for FRET-based sensing in many clinical, environmental and food testing applications nowadays.

Many methods are available for the detection of trace amounts of metal ions, including inductively coupled plasma mass spectrometry (ICP-MS), cold-vapor atomic fluorescence spectroscopy (CV-AFS), and high performance liquid chromatography (HPLC). However, while such techniques offer an excellent sensing performance, they are expensive, bulky, and require professional and technical support. Furthermore, they lack the ability to perform detection in real time. Consequently, the problem of developing simpler and low-cost portable detection systems has attracted great interest in recent years. Labon-a-chip (LOC) devices offer many advantages in this regard, including the ability to perform a wide range of on-chip functions, such as sample preparation and preconcentration [16-18], species mixing [19-23], cell sorting and counting [24-28], polymerase chain reaction (PCR), and so on. Many manufacturing materials and techniques have been proposed for the fabrication of microfluidic devices, including photolithography, laser engraving [29,30] and paper-based devices [31-35]. However, even though lithography has excellent precision and a good miniaturization ability, its production cost is extremely high [13]. Furthermore, while laser engraving has a greatly reduced cost, it induces a high surface roughness and therefore increases the risk of sample blockage in the channels [30]. Similarly, using paper-based devices as a detection platform can also effectively reduce costs and production complexity, but the fibers in the paper may block the sample circulation [15].

Accordingly, the present study employs micro-machining technology (a form of $\mathrm{CNC}$ machining process) to fabricate a microfluidic platform for $\mathrm{Hg}$ (II) and lead $\mathrm{Pb}$ (II) ion detection due to its advantages of speed, a low cost, a simple operation interface, and the ability to manufacture complex structures [36]. The fabricated device has the form of a Ttype micro-mixer incorporating a tortuous flow channel to reduce the distance between the inlet and the outlet and enhance the mixing efficiency. Furthermore, the device is fabricated on PDMS substrates to avoid the presence of residues after cleaning and improve the repeatability of the experimental results accordingly. In the sensing process, a mixed $\mathrm{GO} /$ fluorescent-labeled aptamer solution is injected into the lower inlet (inlet 2) of the device, while a solution containing $\mathrm{Hg}^{2+}$ or $\mathrm{Pb}^{2+}$ ions is injected into the upper inlet (inlet 1) shown in Fig.1. During the subsequent mixing process, the aptamers bond with the $\mathrm{Hg}^{2+}$ or $\mathrm{Pb}^{2+}$ ions to form thymine $(\mathrm{T})$ or guanine $(\mathrm{G})$ complexes, respectively, and the concentration of the ions is detected by observing the corresponding FRET-induced change in 
the fluorescence intensity [12-15,37]. Finally, the detection results are compared with those obtained from a commercial instrument - inductively coupled plasma mass spectrometry (ICP-MS).

\section{Materials and Methods}

\subsection{Materials and instruments}

Graphene oxide (GO) was purchased from Graphenea Inc. (Spain). Nitric acid $\left(\mathrm{HNO}_{3}\right)$, mercury(II) nitrate $\left(\mathrm{Hg}\left(\mathrm{NO}_{3}\right)_{2}\right)$ and lead(II) nitrate $\left(\mathrm{Pb}\left(\mathrm{NO}_{3}\right)_{2}\right)$ were purchased from Japan Pharmaceutical Industry Co., Ltd. (Japan). The other chemicals used in the study: $\mathrm{MgCl}_{2}, \mathrm{Na}_{2} \mathrm{HPO}_{4}, \mathrm{KH}_{2} \mathrm{PO}_{4}, \mathrm{HCL}, \mathrm{NaOH}$ were obtained from Sigma-Aldrich $\mathrm{Co}$ (USA), $\mathrm{NaCl}, \mathrm{KCl}$ and $\mathrm{CuSO}_{4}$ were obtained from J.T. Baker Chemical Co (USA), $\mathrm{CaCl}_{2}$ and $\mathrm{CoCl}_{2}$ were obtained from Panreac (Spain). Poly-dimethylsiloxane (PDMS) was purchased from Sil-More Industrial Ltd. (Taiwan). The solvents were prepared using ultrapure water $(18.2 \mathrm{M} \Omega)$. All of the chemicals were of an analytical grade and were used as-received without further purification. Two DNA probes modified with FAM and HEX fluorescent dye at the $3^{\prime}$ end, respectively, were purchased from Protech Technology Enterprise Co., Ltd. (Taiwan). Aptamer solutions were prepared by dissolving the DNA probes in $100 \mathrm{mM}$ of PBS (pH 7.4). The sequences of the two aptamer probes were as follows:

Probe for $\mathrm{Hg}^{2+}: 5^{\prime}$-TTCTTTCTTCGCGTTGTTTGTT-FAM-3'

Probe for $\mathrm{Pb}^{2+}: 5^{\prime}$-GGAAGGTGTGGAAGG-HEX-3'

The microfluidic device was fabricated using an EGX-400 Professional Rotary Engraver (Roland, Japan) and a PDMS replication process. The mixing efficiency was evaluated using an ECLIPSE Ti Inverted research microscope (Nikon, Japan). In addition, the fluorescence measurements were obtained using a LSM780 scanning laser confocal microscope (Zeiss, Germany).

\subsection{Evaluation of mixing efficiency}

Mixing between two fluids is an essential step in microfluidic devices for enhancing different species mixed and their subsequent reaction. There are many techniques available to achieve such purpose proposed in the literature [19-23]. We design a simple passive mixer with cured flow path in this study. Figure 1(a) illustrates the geometry of the microfluidic channel in the proposed sensing device. A mold was first fabricated in PMMA using the EGX-400 engraver and a replication process was then performed to transfer the microchannel to a PDMS substrate. Finally, the substrate was bonded to a glass slide using oxygen plasma treatment to form the final microfluidic assembly (see Fig. 1(b)). The microchannel has two inlets, each one has $10 \mathrm{~mm}$ length, and the distance of the tortuous microchannel from location \#1 to the outlet is $87 \mathrm{~mm}$. The channel width and height are $0.6 \mathrm{~mm}$ and $0.15 \mathrm{~mm}$, respectively.

The mixing performance of the device was illustrated by injecting blue ink and DI water into the upper and lower inlets, respectively, and then calculating the mixing efficiency at each of the seven locations marked in Fig. 1(b) in accordance with

$$
\text { Mixing Index }(M I)=1-\sqrt{\frac{1}{N} \sum_{i=1}^{N}\left(\frac{\mathrm{I}_{i}-I_{\text {mean }}}{I_{\text {mean }}}\right)^{2}},
$$

where $\mathrm{I}_{\mathrm{i}}$ is the intensity value of pixel $\mathrm{i}$, Imean is the mean intensity of the initial images prior to mixing and $N$ is the total number of pixels within the captured image $[19,37,38]$. The value of Ii was analyzed by an ImageJ software [39].

The flow field and the associated concentration of the two fluids in the 3D microchannel were also simulated by solving the Navier-Stokes equations coupled with the NernstPlanck equations a using a commercial software COMSOL Multiphysics [40]. The mixing index (MI) is calculated numerically by the following integration: 


$$
M I=1-\frac{\int_{A}\left|C-C_{i}\right| d A}{\int_{A}\left|C_{0}-C_{i}\right| d A},
$$

where $\mathrm{C}$ is the concentration at a point on a cross-section plane $\mathrm{A}, \mathrm{C}_{i}$ is the concentration under completed mixing state (i.e., $C_{i}=(1+0) / 2=0.5$, value 1 and 0 is the concentration at upper and lower inlet, respectively), $\mathrm{C}_{0}$ is the concentration at inlet (we choose $\mathrm{C}_{0}=1$ in the calculation).

(a)

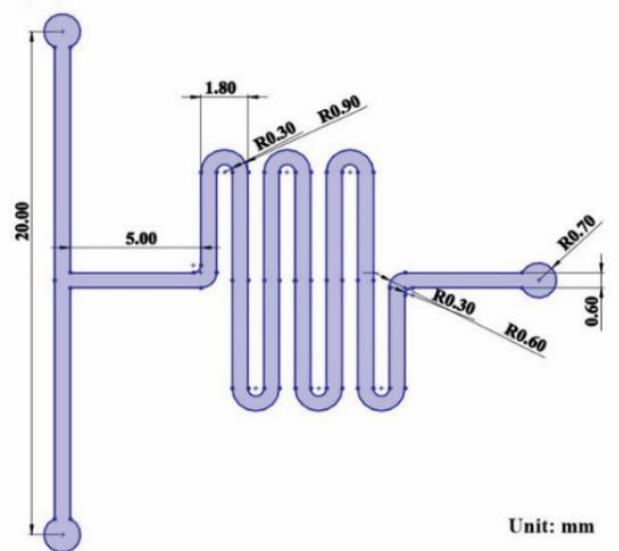

(b)

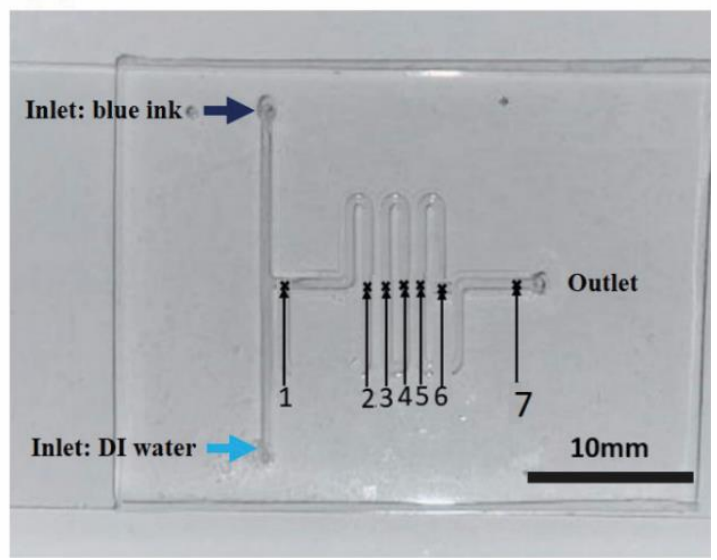

Figure 1. (a) Schematic illustration showing microfluidic device design, and (b) Photograph of actual microfluidic device.

\subsection{Evaluation of quenching efficiency}

The fluorescence quenching efficiency of the GO/aptamer solutions is strongly dependent on the relative concentration of GO. Thus, a series of experiments was performed to measure the fluorescence intensity change of fluorescent-labeled aptamer solutions (100 $\mathrm{nM}$ ) with different concentrations of GO in a glass-bottom dish. For each sample, the quenching efficiency was computed as $\left(\mathbf{f}_{0}-\mathbf{f}\right) / \mathbf{f}_{0}$, where $\mathbf{f}_{0}$ is the original aptamer fluorescence intensity and $\mathbf{f}$ is the measured fluorescence intensity in the presence of both aptamer and GO.

\subsection{Experimental setup}

Figure 2 presents a photograph of the experimental setup. The metal ion solutions and GO/aptamer suspensions were injected into the upper and lower inlet channels of the microfluidic device, respectively, by dedicated syringe pumps and underwent mixing in the tortuous microchannel. The resulting change in the fluorescence intensity of the GO/aptamer suspension was observed at Point \#7 in the microfluidic channel (see Fig. 1(b)) and the quenching effect was then evaluated by image-analysis software installed on an interfaced PC in accordance with (F - Fo) / F, where F is the measured fluorescence intensities at the observed point [41]. Fo is the fluorescence intensity under fully quenched by $\mathrm{GO}$ and its ideal value is 0 . 
(a)

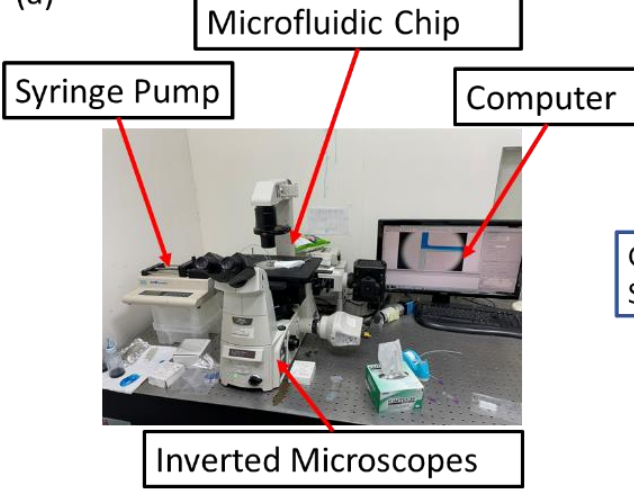

(b)

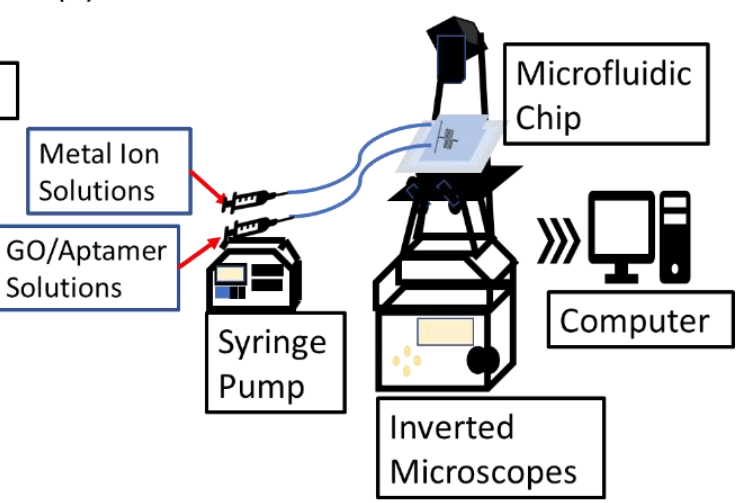

Figure 2. (a) Experimental setup used for $\mathrm{Hg}^{2+}$ and $\mathrm{Pb}^{2+}$ ion detection. (b) Schematic integration of the measurement system.

\section{Results}

\subsection{Mixing performance of microfluidic device and quenching efficiency of GO/ap- tamer suspensions}

Figure 3(a) shows a top-view of the simulated concentration contour along the microchannel. Figure 3(b) shows the mixing index (MI) at different locations of microchannel as function of flow rate (Q: $\mu \mathrm{L} / \mathrm{min})$. Each experimental data is repeatedly measured three times $(\mathrm{N}=3)$. The numerical results are shown to be well consistent with the experimental results. The results show that the lower flow rates (i.e. below $5 \mu \mathrm{L} / \mathrm{min}$ ) has better mixing indices at location $\# 7$, where the MI are over $95 \%$. The reason is the fluid spends more time to travel through the microchannel for a lower flow rate and the contact/diffusion time between two fluids increase. Therefore, all subsequent experiments conducted in this study is adopting the flow rate at $3 \mu \mathrm{L} / \mathrm{min}$ to ensure the two fluids being well mixed.

(a)

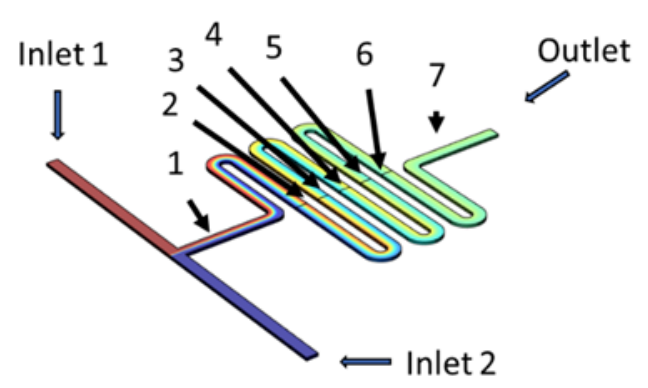

(b)

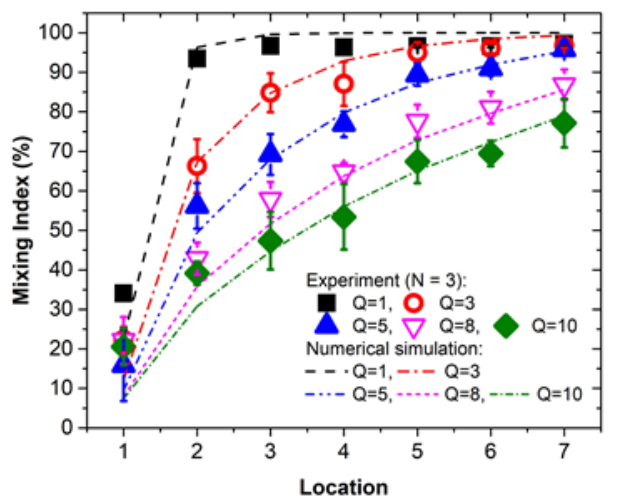

Figure 3. (a) Concentration contour distribution via numerical simulation. (b) Mixing Index (MI) at different locations along the microchannel as function of flow rate (Q: $\mu \mathrm{L} / \mathrm{min})$. Experimental and numerical simulation results mutually agree well. Over $95 \%$ mixing index can be obtained for the flow rated lower than $5 \mu \mathrm{L} / \mathrm{min}$ at the detection location \#7.

The quenching efficiency of the $\mathrm{Hg}^{2+}$ and $\mathrm{Pb}^{2+} \mathrm{GO} /$ aptamer suspensions was evaluated in glass-bottom dishes using various GO concentrations in the range of 1 500 ppm. The results presented in Fig. 4(a) show that for both probes, the quenching efficiency remains approximately constant as the GO concentration is increased beyond $100 \mathrm{ppm}$. Thus, to ensure the complete suppression of the GO concentration effect, an excess concentration of $500 \mathrm{ppm}$ GO was used in all of the remaining experiments unless stated otherwise [42]. The long-term quenching effect of the GO/aptamer suspensions was observed 
in glass-bottom dishes over a period of 400 s. As shown in Fig. 4(b), the fluorescence intensity of both probes gradually reduced over time as a result of the FRET GO quenching effect. From inspection, the quenching efficiencies of the FAM- and HEX-labeled probes stabilized at approximately 0.98 and 0.94 after 180 and 40 seconds, respectively.

(a)

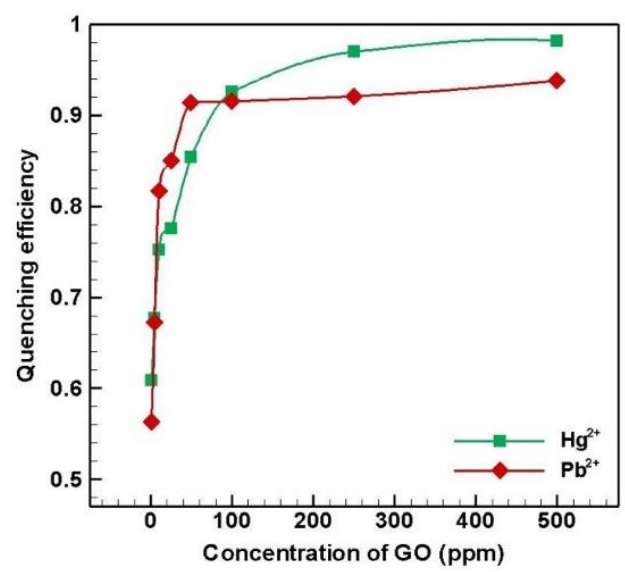

(b)

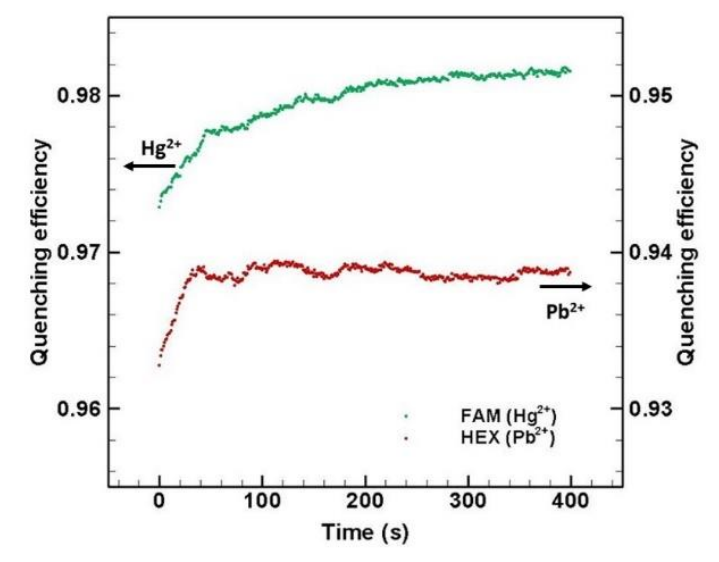

Figure 4. (a)Quenching efficiency for suspensions consisting of FAM- and HEX-labeled $100 \mathrm{nM}$ aptamers and GO solution with concentration ranging from 1 to $500 \mathrm{ppm}$, and (b) Long-term quenching efficiency of suspensions of FAM- and HEX-labeled aptamers and GO solution with concentration of $500 \mathrm{ppm}$.

\subsection{Sensitivity of $\mathrm{Hg}^{2+}$ and $\mathrm{Pb}^{2+}$ detection}

FAM- and HEX-labeled aptamer solutions with a concentration of $100 \mathrm{nM}$ were mixed with 500 ppm GO solution and incubated for 10 minutes to ensure a complete quenching of the aptamers. The suspensions were then injected into the lower inlet of the microfluidic device. Meanwhile, $\mathrm{Hg}^{2+}$ samples with concentrations of $10,25,50,100,250$, 500, 1000 and $2000 \mathrm{nM}$ (approximately 2.0, 5.0, 10.0, 20.1, 50.2, 100.3, 200.6 and $401.2 \mathrm{ppb}$ ) and $\mathrm{Pb}^{2+}$ samples with concentrations of 10, 25, 50, 100, 250, 500, 1000 and $2000 \mathrm{nM}$ (approximately 2.1, 5.2, 10.4, 20.7, 51.8, 103.6, 207.2 and $414.4 \mathrm{ppb}$ ) were injected into the upper inlet of the device. For each injected sample, the resulting change (i.e., increase) in the fluorescence intensity was recorded at location \#7 in the microfluidic channel after $180 \mathrm{~s}$ (FAM-labeled probe) or $40 \mathrm{~s}$ (HEX-labeled probe). The corresponding results are presented in Figs. 5(a) and 5(b) for the $\mathrm{Hg}^{2+}$ and $\mathrm{Pb}^{2+}$ probes, respectively. For both probes, the fluorescence intensity remains approximately constant as the metal ion content increases beyond $500 \mathrm{nM}$. However, the fluorescence intensity increases linearly with the $\mathrm{Hg}^{2+}$ concentration from 10 to $250 \mathrm{nM}\left(\mathrm{R}^{2}=0.95845\right)$ and with the $\mathrm{Pb}^{2+}$ concentration from 10 to $100 \mathrm{nM}\left(\mathrm{R}^{2}=0.95923\right)$. The corresponding correlation relationships have the form of $\mathrm{Y}=0.00092 \mathrm{X}+0.51459$ and $\mathrm{Y}=0.00208 \mathrm{X}+0.57557$, respectively. 
(a)

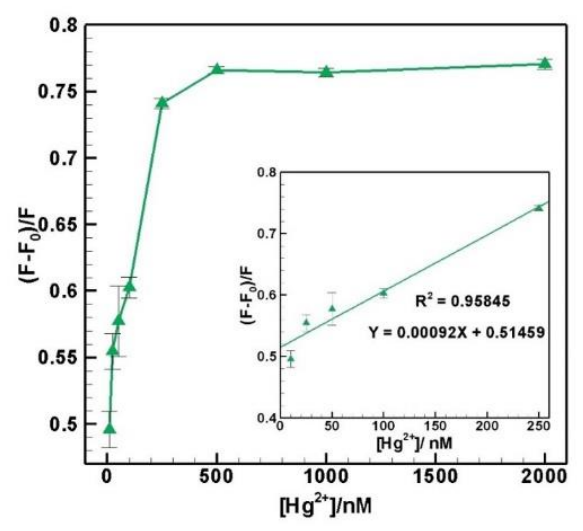

(b)

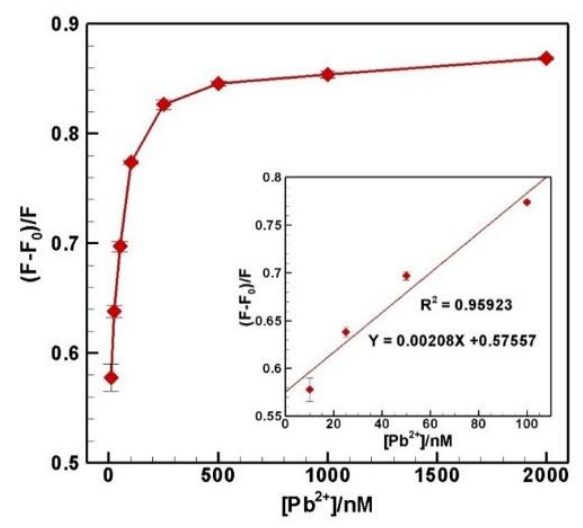

Figure 5. (a)Fluorescence intensity recovery following mixing with $\mathrm{Hg}^{2+}$ solutions with concentrations ranging from 10 to $2000 \mathrm{nM}$ ( $500 \mathrm{ppm}$ GO). Insert: calibration curve for $\mathrm{Hg}^{2+}$ solutions with concentrations ranging from 10 to $250 \mathrm{nM}$, and (b) Fluorescence intensity recovery following mixing with $\mathrm{Pb}^{2+}$ solutions with concentrations ranging from 10 to $2000 \mathrm{nM}$ (500 ppm GO). Insert: calibration curve for $\mathrm{Pb}^{2+}$ solutions with concentrations ranging from 10 to $100 \mathrm{nM}$.

The results show that the fluorescence intensity increases linearly with an increasing $\mathrm{Hg}^{2+}$ and $\mathrm{Pb}^{2+}$ concentration over the ranges of $10 \sim 250 \mathrm{nM}$ (i.e., 2.0 50 ppb) and 10 100 nM (i.e., 2.1 20.7 ppb), respectively. Moreover, the limits of detection (LoDs) for $\mathrm{Hg}^{2+}$ and $\mathrm{Pb}^{2+}$ are equal to $2.01 \mathrm{ppb}$ and $2.07 \mathrm{ppb}$, respectively. The results show that the sensing platform provides a feasible solution for enforcing the WHO recommendation of no more than 6

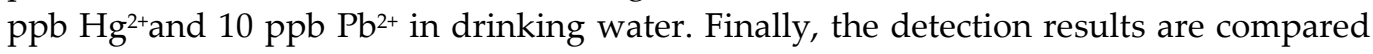
well with those obtained from a commercial instrument by inductively coupled plasma mass spectrometry (ICP-MS) as shown in Fig.6. Excellent agreement between the results obtained by the present microfluidic device and the ICP-MS is demonstrated.

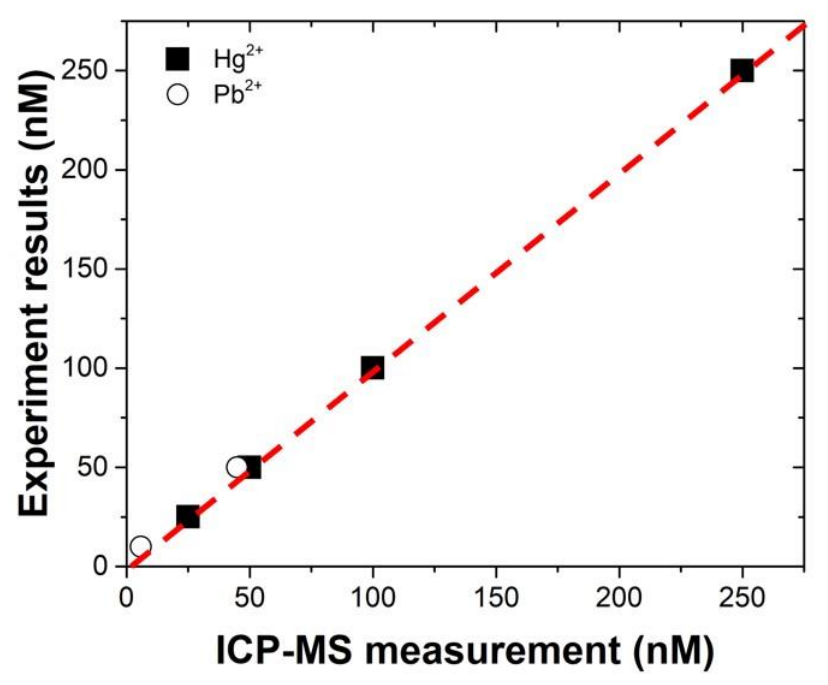

Figure 6. Comparison of results obtained by the ICP-MS and the present microfluidic device.

\subsection{Selectivity of $\mathrm{Hg}^{2+}$ and $\mathrm{Pb}^{2+}$ detection}

To test the selectivity of the proposed device, GO/aptamer solutions were injected into the lower inlet of the microfluidic device, while mixed $\mathrm{Hg}^{2+}$ and $\mathrm{Pb}^{2+}$ samples with concentration 10, 100 and $1000 \mathrm{nM}$, respectively, were injected into the upper inlet. The corresponding fluorescence intensity measurements are shown in Fig. 7(a). For both 
probes, the fluorescence intensity shows a good recovery performance for all values of the concentration (i.e., low, medium and high). In other words, even though the fluorescence recovery reduces as the concentration of the competing metal ions increases, the device still retains the ability to discriminate the target ions. In addition, control experiments were performed using several other bivalent metal ions, namely $\mathrm{Co}^{2+}, \mathrm{Ca}^{2+}, \mathrm{Mg}^{2+}$ and $\mathrm{Cu}^{2+}$ ions. Figure 7(b) shows the detection results obtained for mixed $10 \mathrm{nM} \mathrm{Hg}^{2+}$ and $\mathrm{Pb}^{2+}$ solutions containing an additional $10 \mu \mathrm{M}$ of $\mathrm{Co}^{2+}, \mathrm{Ca}^{2+}, \mathrm{Mg}^{2+}$ and $\mathrm{Cu}^{2+}$ ions, respectively. It is seen that the competing ions have only a minor effect on the measured fluorescence change despite their relatively high concentration. In other words, the selectivity of the proposed sensor for $\mathrm{Hg}^{2+}$ and $\mathrm{Pb}^{2+}$ ion detection is further confirmed.

(a)

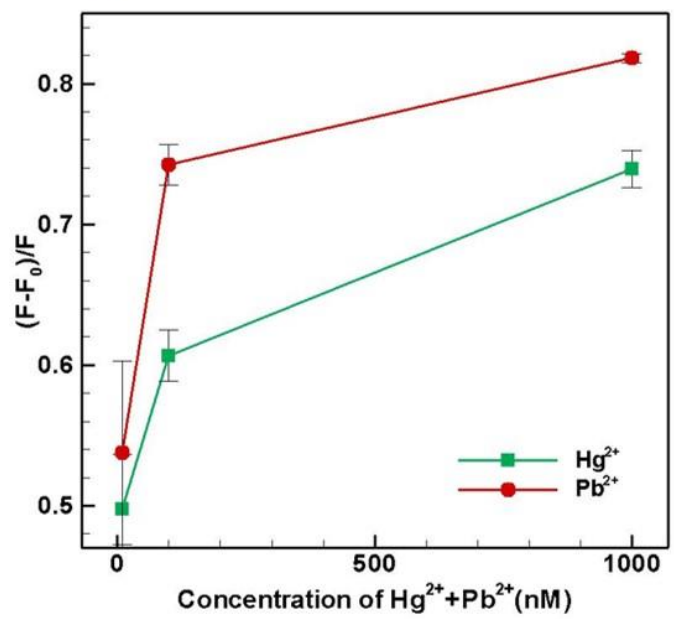

(b)

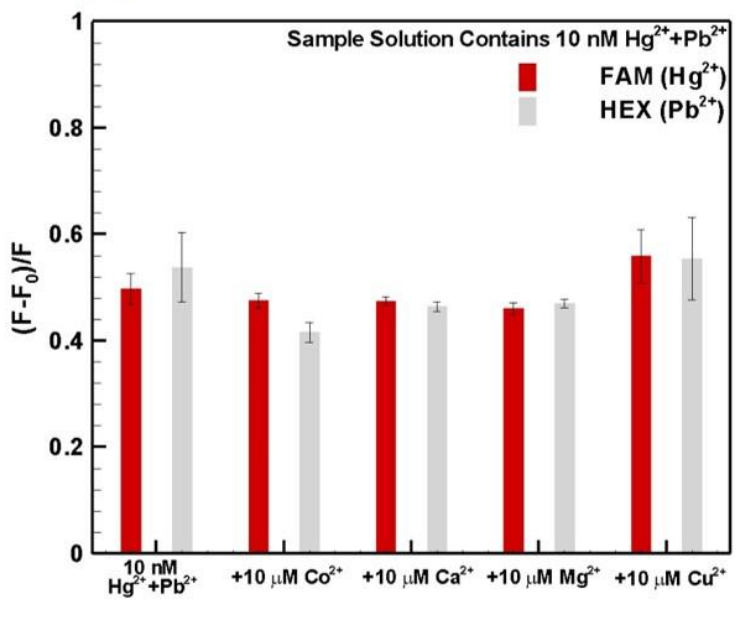

Figure 7. Fluorescence intensity recovery for competitive assays involving: (a) different concentration of $\mathrm{Hg}^{2+}$ and $\mathrm{Pb}^{2+}$, and (b) $10 \mu \mathrm{M} \mathrm{Co}^{2+}, \mathrm{Ca}^{2+}, \mathrm{Mg}^{2+}$ and $\mathrm{Cu}^{2+}$, respectively (500 ppm GO).

\section{Conclusions}

This study has proposed two FRET-based GO/aptamer sensors for the detection of $\mathrm{Hg}^{2+}$ and $\mathrm{Pb}^{2+}$ ions, respectively, in a PDMS microfluidic device. In the proposed approach, two specifically-chosen aptamer sequences are labeled with FAM and HEX fluorescent dye for visualization purposes and are then quenched by GO. Following interaction between the labeled aptamers and the $\mathrm{Hg}^{2+}$ and $\mathrm{Pb}^{2+}$ ions, respectively, a discernable restoration of the fluorescent intensity occurs as a result of the FRET effect. The change in the fluorescent intensity is then used to inversely derive the corresponding $\mathrm{Hg}^{2+}$ and $\mathrm{Pb}^{2+}$ concentration. Sensing experiments have been performed using $\mathrm{Hg}^{2+}$ and $\mathrm{Pb}^{2+}$ solutions with concentrations of 10, 25, 50, 100, 250, 500, 1000 and $2000 \mathrm{nM}$ (i.e., $2 \sim 400 \mathrm{ppb}$ ), respectively. The results have shown that the fluorescence intensity increases linearly with an increasing $\mathrm{Hg}^{2+}$ and $\mathrm{Pb}^{2+}$ concentration over the ranges of 10 250 $\mathrm{nM}$ (i.e., 2.0 50 ppb) and 10 100 $\mathrm{nM}$ (i.e., 2.1 20.7 ppb), respectively. Moreover, the limits of detection (LoDs) for $\mathrm{Hg}^{2+}$ and $\mathrm{Pb}^{2+}$ are equal to $2.01 \mathrm{ppb}$ and $2.07 \mathrm{ppb}$, respectively. Finally, the experimental results obtained for competitive assays involving $\mathrm{Hg}^{2+}$ and $\mathrm{Pb}^{2+}$ ions and other metal ions $\left(\mathrm{Co}^{2+}, \mathrm{Ca}^{2+}\right.$, $\mathrm{Mg}^{2+}$ and $\mathrm{Cu}^{2+}$ ) have shown that the proposed device has good selectively. Overall, the results show that the proposed sensing device and platform provides a feasible solution

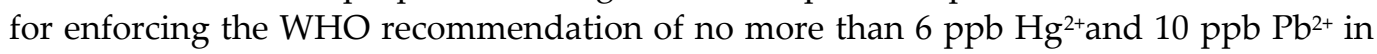
drinking water.

Author Contributions: Conceptualization, Wei-Hao Huang, Ruo-Yin Wu and Ruey-Jen Yang; Data curation and Methodology, Wei-Hao Huang and Ruo-Yin Wu; Writing-original draft, Wei-Hao Huang and Ruo-Yin Wu; Writing - review \& editing, Ruey-Jen Yang; Funding acquisition, Ruey-Jen Yang; Simulation and experiment, Ruo-Yin Wu, Ko-Li Yeh and Van-Phung Mai; Project administration, Ruey-Jen Yang. All authors have read and agreed to the published version of the manuscript. 
Acknowledgments: The authors gratefully acknowledge the financial support provided to this study by the Ministry of Science and Technology (MOST) of Taiwan under Project No. 107-2221-E006-127-MY3.

Conflicts of Interest: The authors declare that they have no known competing financial interests or personal relationships that could have appeared to influence the work reported in this paper. 


\section{References}

1. Duruibe, J.O.; Ogwuegbu, M.; Egwurugwu, J. Heavy metal pollution and human biotoxic effects. International Journal of Physical Sciences 2007, 2, 112-118.

2. Zahir, F.; Rizwi, S.J.; Haq, S.K.; Khan, R.H. Low dose mercury toxicity and human health. Environmental Toxicology and Pharmacology 2005, 20, 351-360.

3. Papanikolaou, N.C.; Hatzidaki, E.G.; Belivanis, S.; Tzanakakis, G.N.; Tsatsakis, A.M. Lead toxicity update. A brief review. Medical Science Monitor 2005, 11, RA329-RA336.

4. Wang, L.; Peng, X.; Fu, H.; Huang, C.; Li, Y.; Liu, Z. Recent advances in the development of electrochemical aptasensors for detection of heavy metals in food. Biosensors and Bioelectronics 2020, 147, 111777.

5. Khan, N.I.; Song, E. Lab-on-a-chip systems for aptamer-based biosensing. Micromachines 2020, 11, 220.

6. Ono, A.; Togashi, H. Highly selective oligonucleotide - based sensor for mercury (II) in aqueous solutions. Angewandte Chemie 2004, 116, 4400-4402.

7. Li, X.; Wang, G.; Ding, X.; Chen, Y.; Gou, Y.; Lu, Y. A “turn-on” fluorescent sensor for detection of Pb ${ }^{2+}$ based on graphene oxide and G-quadruplex DNA. Physical Chemistry Chemical Physics 2013, 15, 12800-12804.

8. Ran, G.; Wu, F.; Ni, X.; Li, X.; Li, X.; Liu, D.; Sun, J.; Xie, C.; Yao, D.; Bai, W. A novel label-free electrochemical aptasensor with one-step assembly process for rapid detection of lead (II) ions. Sensors and Actuators B: Chemical 2020, $320,128326$.

9. Baghayeri, M.; Amiri, A.; Maleki, B.; Alizadeh, Z.; Reiser, O. A simple approach for simultaneous detection of cadmium (II) and lead (II) based on glutathione coated magnetic nanoparticles as a highly selective electrochemical probe. Sensors and Actuators B: Chemical 2018, 273, 1442-1450.

10. Liu, C.-W.; Tsai, T.-C.; Osawa, M.; Chang, H.-C.; Yang, R.-J. Aptamer-based sensor for quantitative detection of mercury (II) ions by attenuated total reflection surface enhanced infrared absorption spectroscopy. Analytica Chimica Acta 2018, 1033, 137-147.

11. Hussain, S.A. An introduction to fluorescence resonance energy transfer (FRET). Science Journal of Physics 2012, $268,4$.

12. Matte, H.R.; Subrahmanyam, K.; Rao, K.V.; George, S.J.; Rao, C. Quenching of fluorescence of aromatic molecules by graphene due to electron transfer. Chemical Physics Letters 2011, 506, 260-264.

13. Park, M.; Seo, T.S. An integrated microfluidic device with solid-phase extraction and graphene oxide quantum dot array for highly sensitive and multiplex detection of trace metal ions. Biosensors and Bioelectronics 2019, 126, 405-411.

14. Dong, R.; Liu, Y.; Mou, L.; Deng, J.; Jiang, X. Microfluidics - based biomaterials and biodevices. Advanced Materials 2019, 31, 1805033.

15. Bian, R.-X.; Wu, X.-T.; Chai, F.; Li, L.; Zhang, L.-Y.; Wang, T.-T.; Wang, C.-G.; Su, Z.-M. Facile preparation of fluorescent Au nanoclusters-based test papers for recyclable detection of $\mathrm{Hg}^{2+}$ and $\mathrm{Pb}^{2+}$. Sensors and Actuators B: Chemical 2017, 241, 592-600.

16. Fu, L.M.; Hou, H.H.; Chiu, P.H.; Yang, R.J. Sample preconcentration from dilute solutions on micro/nanofluidic platforms: A review. Electrophoresis 2018, 39, 289-310.

17. Yang, R.-J.; Pu, H.-H.; Wang, H.-L. Ion concentration polarization on paper-based microfluidic devices and its application to preconcentrate dilute sample solutions. Biomicrofluidics 2015, 9, 014122.

18. Hung, L.-H.; Wang, H.-L.; Yang, R.-J. A portable sample concentrator on paper-based microfluidic devices. Microfluid. Nanofluid. 2016, 20, 80.

19. Chang, C.; Yang, R.-J. Computational analysis of electrokinetically driven flow mixing in microchannels with patterned blocks. Journal of Micromechanics and Microengineering 2004, 14, 550.

20. Tai, C.H.; Yang, R.J.; Huang, M.Z.; Liu, C.W.; Tsai, C.H.; Fu, L.M. Micromixer utilizing electrokinetic instability - induced shedding effect. Electrophoresis 2006, 27, 4982-4990.

21. Chen, J.K.; Yang, R.J. Electroosmotic flow mixing in zigzag microchannels. Electrophoresis 2007, 28, 975-983.

22. Chang, C.-C.; Yang, R.-J. Chaotic mixing in a microchannel utilizing periodically switching electro-osmotic recirculating rolls. Physical Review E 2008, 77, 056311. 
23. Chen, J.-K.; Weng, C.-N.; Yang, R.-J. Assessment of three AC electroosmotic flow protocols for mixing in microfluidic channel. Lab on a Chip 2009, 9, 1267-1273.

24. Yang, R.-J.; Fu, L.-M.; Hou, H.-H. Review and perspectives on microfluidic flow cytometers. Sensors and Actuators B: Chemical 2018, 266, 26-45.

25. Fu, L.-M.; Yang, R.-J.; Lin, C.-H.; Pan, Y.-J.; Lee, G.-B. Electrokinetically driven micro flow cytometers with integrated fiber optics for on-line cell/particle detection. Analytica Chimica Acta 2004, 507, 163-169.

26. Lee, G.-B.; Chang, C.-C.; Huang, S.-B.; Yang, R.-J. The hydrodynamic focusing effect inside rectangular microchannels. Journal of Micromechanics and Microengineering 2006, 16, 1024.

27. Chang, C.-C.; Huang, Z.-X.; Yang, R.-J. Three-dimensional hydrodynamic focusing in two-layer polydimethylsiloxane (PDMS) microchannels. Journal of Micromechanics and Microengineering 2007, 17, 1479.

28. Lee, H.-C.; Hou, H.-H.; Yang, R.-J.; Lin, C.-H.; Fu, L.-M. Microflow cytometer incorporating sequential micro-weir structure for three-dimensional focusing. Microfluidics and Nanofluidics 2011, 11, 469-478.

29. Fu, L.-M.; Ju, W.-J.; Yang, R.-J.; Wang, Y.-N. Rapid prototyping of glass-based microfluidic chips utilizing two-pass defocused $\mathrm{CO}_{2}$ laser beam method. Microfluidics and Nanofluidics 2013, 14, 479-487.

30. Li, X.; Yuan, G.; Yu, W.; Xing, J.; Zou, Y.; Zhao, C.; Kong, W.; Yu, Z.; Guo, C. A self-driven microfluidic surface-enhanced Raman scattering device for $\mathrm{Hg}^{2+}$ detection fabricated by femtosecond laser. Lab on a Chip 2020, 20, 414-423.

31. Chen, G.-H.; Chen, W.-Y.; Yen, Y.-C.; Wang, C.-W.; Chang, H.-T.; Chen, C.-F. Detection of mercury (II) ions using colorimetric gold nanoparticles on paper-based analytical devices. Analytical Chemistry 2014, 86, 6843-6849.

32. Chen, X.; Mo, D.; Gong, M. A flexible method for nanofiber-based 3D microfluidic device fabrication for water quality monitoring. Micromachines 2020, 11, 276.

33. Yang, R.-J.; Tseng, C.-C.; Ju, W.-J.; Wang, H.-L.; Fu, L.-M. A rapid paper-based detection system for determination of human serum albumin concentration. Chemical Engineering Journal 2018, 352, 241-246.

34. Yang, R.-J.; Tseng, C.-C.; Ju, W.-J.; Fu, L.-M.; Syu, M.-P. Integrated microfluidic paper-based system for determination of whole blood albumin. Sensors and Actuators B: Chemical 2018, 273, 1091-1097.

35. Tseng, C.-C.; Yang, R.-J.; Ju, W.-J.; Fu, L.-M. Microfluidic paper-based platform for whole blood creatinine detection. Chemical Engineering Journal 2018, 348, 117-124.

36. Chen, P.-C.; Pan, C.-W.; Lee, W.-C.; Li, K.-M. An experimental study of micromilling parameters to manufacture microchannels on a PMMA substrate. The International Journal of Advanced Manufacturing Technology 2014, 71, 1623-1630. Jain, R.; Thakur, A.; Kaur, P.; Kim, K.-H.; Devi, P. Advances in imaging-assisted sensing techniques for heavy metals in water: Trends, challenges, and opportunities. TrAC Trends in Analytical Chemistry 2020, 123, 115758.

38. Wang, H.-L.; Chu, C.-H.; Tsai, S.-J.; Yang, R.-J. Aspartate aminotransferase and alanine aminotransferase detection on paperbased analytical devices with inkjet printer-sprayed reagents. Micromachines 2016, 7, 9.

39. Pérez, J.M.M.; Pascau, J. Image processing with ImageJ; Packt Publishing Ltd: 2013.

40. COMSOL Multiphysics@ (COMSOL AB, Sweden). https://www.comsol.com.

41. Zhan, S.; Wu, Y.; Luo, Y.; Liu, L.; He, L.; Xing, H.; Zhou, P. Label-free fluorescent sensor for lead ion detection based on lead (II)-stabilized G-quadruplex formation. Analytical Biochemistry 2014, 462, 19-25.

42. Wu, X.; Xing, Y.; Zeng, K.; Huber, K.; Zhao, J.X. Study of fluorescence quenching ability of graphene oxide with a layer of rigid and tunable silica spacer. Langmuir 2018, 34, 603-611. 